\title{
CORRIGENDUM
}

\section{Evolution and obesity: resistance of obese-prone rats to a challenge of food restriction and wheel running}

\author{
WD Pierce, A Diane, CD Heth, JC Russell and SD Proctor
}

International Journal of Obesity (2010) 34, 1665; doi:10.1038/ijo.2010.115

Correction to: International Journal of Obesity (2010) 34, 589-592; doi:10.1038/ijo.2009.294; published online 12 January 2010

After the publication of the article, the authors noticed an error in Table 1: the values for corticosterone in Table 1 were calculated as $\mathrm{nmol}^{-1}$ rather than $\mathrm{pmoll}^{-1}$.
The correct table is reproduced below.

The authors would like to apologize for this mistake.

Table 1 Data are the biochemical parameters before and after the challenge

\begin{tabular}{|c|c|c|c|c|c|}
\hline \multirow[t]{2}{*}{ Biochemical parameters } & \multicolumn{2}{|c|}{ Juveniles rats } & \multicolumn{2}{|c|}{ Adolescent rats } & \multirow{2}{*}{$\begin{array}{l}\text { ANOVA } \\
\text { Effect }^{\mathrm{a}}\end{array}$} \\
\hline & Lean prone & Obese prone & Lean prone & Obese prone & \\
\hline \multicolumn{6}{|l|}{ Before challenge } \\
\hline Triglycerides $\left(\mathrm{mmoll}^{-1}\right)$ & $2.59 \pm 0.41_{\mathrm{a}}^{\mathrm{b}}$ & $9.74 \pm 0.88_{b}^{b}$ & $3.36 \pm 0.38_{\mathrm{a}}^{\mathrm{b}}$ & $21.56 \pm 3.29_{b}^{b}$ & $G, A, G \times A$ \\
\hline Leptin $\left(p g \mathrm{ml}^{-1}\right)$ & $2.4 \pm 1.1_{\mathrm{a}}$ & $235 \pm 16_{\mathrm{b}^{\mathrm{b}}}^{\mathrm{b}}$ & $2.6 \pm 1.0_{a}$ & $429 \pm 30_{b}^{b}$ & $G, A, G \times A$ \\
\hline Insulin $\left(\mathrm{pg} \mathrm{ml}^{-1}\right)$ & $34.2 \pm 4.5^{a}{ }^{b}$ & $585 \pm 13_{b}$ & $35 \pm 5_{\mathrm{a}}^{\mathrm{b}}$ & $101 \pm 13_{b}$ & $\mathrm{G}, \mathrm{A}$ \\
\hline Glucose $\left(\mathrm{mmoll}^{-1}\right)$ & $5.31 \pm 0.16_{\mathrm{a}}^{\mathrm{b}}$ & $5.72 \pm 0.41_{\mathrm{a}}$ & $5.38 \pm 0.26_{a}$ & $6.06 \pm 0.27 \mathrm{a}$ & NS \\
\hline \multicolumn{6}{|l|}{ After challenge } \\
\hline Triglycerides $\left(\mathrm{mmolI}^{-1}\right)$ & $0.59 \pm 0.14_{a}$ & $6.3 \pm 0.59_{b}$ & $0.89 \pm 0.29 \mathrm{a}$ & $7.36 \pm 0.64_{b}$ & G \\
\hline Leptin $\left(\mathrm{pg} \mathrm{ml}^{-1}\right)$ & ND & $105 \pm 16$ & ND & $185 \pm 38$ & \\
\hline Insulin $\left(\mathrm{pg} \mathrm{ml}^{-1}\right)$ & $11 \pm 1.3_{a}$ & $270 \pm 85_{b}$ & $8.8 \pm 1.8_{\mathrm{a}}$ & $672 \pm 369_{b}$ & G \\
\hline Glucose $\left(\mathrm{mmoll} \mathrm{I}^{-1}\right)$ & $4.23 \pm 0.43_{a}$ & $7.36 \pm 0.83_{a}$ & $4.62 \pm 0.52_{a}$ & $6.39 \pm 0.51_{a}$ & G \\
\hline Corticosterone $\left(\mathrm{nmolI}^{-1}\right)$ & $1067 \pm 23_{a}$ & $476 \pm 107_{b}$ & $936 \pm 82_{a}$ & $591 \pm 103_{b}$ & G \\
\hline ACTH $\left(\mathrm{pg} \mathrm{ml}^{-1}\right)$ & $347 \pm 89 a$ & $223 \pm 18 \mathrm{a}$ & $208 \pm 46_{a}$ & $130 \pm 90_{a}$ & NS \\
\hline
\end{tabular}

Abbreviations: A, main effect of age; ACTH, adenocorticotropin hormone; ANOVA, analysis of variance; $\mathrm{G} \times \mathrm{A}$, interaction of genotype and age; $\mathrm{G}$, main effect of genotype; ND, not detectable; NS, not significant. The values are the means and standard errors of the mean (s.e.m.) for each age (juvenile vs adolescent) by genotype (lean-prone vs obese-prone) group $(n=6)$ of JCR-LA-cp rats. Results of ANOVAs are also shown. All tests were conducted with $\alpha$ set at 0.05 level. For the same age group, means with different subscripts are statistically different. ${ }^{a}$ Main and interaction effects for ANOVAs with age (juvenile, adolescent) and genotype $(c p / c p,+/ ?)$. Only significant effects are reported. ${ }^{b}$ Within the same column the measures before and after the challenge are significantly different. 